\title{
Barriers to Guideline-Based Use of Proton Pump Inhibitors to Prevent Upper Gastrointestinal Bleeding
}

\author{
Jacob E. Kurlander, $M D, M S^{1,2,3}$ \\ Danielle Helminski, MPH \\ Alex N. Kokaly, MD, MHSA \\ Caroline R. Richardson, $M D^{2,5}$ \\ Raymond De Vries, $\mathrm{PbD}^{2,6,7}$ \\ Sameer D. Saini, MD, $M S^{1,2,3}$ \\ Sarab L. Krein, PbD, $\mathrm{RN}^{1,2,3}$
}

'Department of Internal Medicine, University of Michigan, Ann Arbor, Michigan

${ }^{2}$ Institute for Healthcare Policy and Innovation, University of Michigan, Ann Arbor, Michigan

${ }^{3}$ Veterans Affairs Ann Arbor Center for Clinical Management Research, Ann Arbor, Michigan

${ }^{4}$ UCLA Health Department of Medicine, Los Angeles, California

${ }^{5}$ Department of Family Medicine, University of Michigan, Ann Arbor, Michigan

${ }^{6}$ Department of Learning Health Sciences, University of Michigan, Ann Arbor, Michigan

${ }^{7}$ Center for Bioethics and Social Sciences in Medicine, University of Michigan, Ann Arbor Michigan

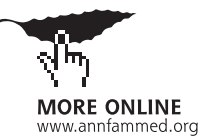

Conflicts of interest: authors report none.

\section{CORRESPONDING AUTHOR}

Jacob E. Kurlander 3912 Taubman Center 1500 E. Medical Center Drive, SPC 5362

Ann Arbor, MI 48109

jkurland@umich.edu

\begin{abstract}
PURPOSE Gastrointestinal (GI) bleeding is one of the most common serious adverse drug events. Guidelines recommend proton pump inhibitor (PPI) gastroprotection to prevent upper Gl bleeding in high-risk patients, but this practice is underused.
\end{abstract}

METHODS To explore prescribing practices and barriers to the use of PPI gastroprotection, including dynamics within and across specialties, we conducted semistructured interviews with physicians in 4 specialties at a single institution. We performed thematic analysis of barriers, organized around the theoretical domains framework.

RESULTS The sample included 5 primary care physicians (PCPs), 4 cardiologists, 3 gastroenterologists, and 3 vascular surgeons. Most PCPs, gastroenterologists, and vascular surgeons seldom prescribed PPI gastroprotection. Cardiologists varied most in their use of PPI gastroprotection, with some prescribing it consistently and others never. Major barriers related to the following 3 themes: (1) knowledge, (2) decision processes, and (3) professional role. Knowledge of guidelines was greatest among cardiologists and gastroenterologists and low among PCPs and vascular surgeons, and PCPs tended to focus on adverse effects associated with PPIs, which made them reluctant to prescribe them. For cardiologists, prevention of bleeding was usually a priority, but they sometimes deferred prescribing to others. For the other 3 specialties, PPI gastroprotection was a low priority. There was unclear delineation of responsibility for prescribing gastroprotection between specialties.

CONCLUSIONS Major barriers to PPI gastroprotection relate to knowledge, decision processes, and professional role, which operate differentially across specialties. Multicomponent interventions will likely be necessary to improve guideline-based use of PPIs to prevent upper GI bleeding.

\section{VISUAL ABSTRACT}

Ann Fam Med 2022;20:5-11. https://doi.org/10.1370/afm.2734.

\section{INTRODUCTION}

$\mathrm{N}$ onvariceal upper gastrointestinal bleeding (UGIB) affects nearly onequarter of a million patients in the United States annually and carries a $2 \%$ case fatality rate. ${ }^{1,2}$ In the United States, the most common cause of UGIB is acid peptic disease including esophageal, gastric, and duodenal ulcers. ${ }^{3}$ Upper gastrointestinal bleeding is often iatrogenic ${ }_{i}$ commonly used medications, including aspirin, nonaspirin nonsteroidal anti-inflammatory drugs (NSAIDs), thienopyridines, and anticoagulants ("high-risk drugs" hereafter) increase the risk. ${ }^{4}$ In fact, warfarin and oral antiplatelet drugs are the first and third most common drugs implicated in emergency hospitalization for adverse drug events, with gastrointestinal bleeding being the single most common manifestation. ${ }^{5}$

Proton pump inhibitors (PPIs) have been proven to decrease the risk of UGIB by $79 \%$ among patients using aspirin or NSAIDs, according to a meta-analysis of 18 randomized controlled trials. ${ }^{6}$ Accordingly, numerous guidelines, endorsed by gastroenterology and cardiology professional organizations, recommend "PPI gastroprotection" for patients at increased risk. Patients commonly at high risk include those who use 2 or more antithrombotic drugs, those aged $>65$ years using NSAIDs (especially at high dose), and those with a history of UGIB who use any single high-risk drug (refer to the guidelines for other particular high-risk groups). ${ }^{7.13}$ On the basis of randomized trial data, for high-risk NSAID users the number needed to treat to prevent asymptomatic ulcers, symptomatic ulcers, and 
upper gastrointestinal (GI) bleeding is roughly 5, 20, and 100, respectively, although benefit varies substantially according to individual risk factors including age and comorbid malignancy, renal disease, and liver disease. ${ }^{14}$ Yet, a minority of patients at high risk for UGIB receive PPIs. In a systematic review of 23 observational studies, the median level of appropriate gastroprotection in high-risk elderly patients taking NSAIDs was $24 \% .^{15}$

Reasons for the underuse of PPI gastroprotection have not been fully elucidated. Knowledge of indications for PPI gastroprotection might be one potential barrier. Prescribing decisions for PPIs have also become more complicated because of observational studies linking PPI use with bone fractures, Clostridioides difficile infection, and other conditions, ${ }^{16}$ which have caused many primary care physicians (PCPs) to change how they use PPIs. ${ }^{17}$ Another unexplored possibility is lack of "ownership" for gastroprotection, given that PCPs and other specialists often comanage patients who are appropriate for gastroprotection.

Understanding barriers to the use of PPI gastroprotection is essential for designing effective interventions to address this issue. The present qualitative study aimed to characterize prescribing practices and barriers to the guideline-based use of PPI gastroprotection among physicians in multiple specialties and the dynamics of PPI prescribing across medical specialties.

\section{METHODS \\ Design and Participants}

We conducted semistructured interviews in person and by telephone with physicians in primary care, cardiology, gastroenterology, and vascular surgery at an integrated, tertiary, academic medical center in the Midwestern United States during the period July to December 2019. We used purposive sampling to include clinicians in each specialty to gain a diverse range of perspectives. We selected these specialties because they all treat patients appropriate for PPI gastroprotection. Eligible clinicians (15 PCPs, 7 cardiologists, 6 gastroenterologists, and 4 vascular surgeons) were invited to participate via e-mail. We continued interviewing new participants until data saturation, the point at which interviews in each specialty yielded little novel information, was reached. ${ }^{18}$ Data were gathered as part of the development process for an intervention to improve appropriate use of PPI gastroprotection.

\section{Data Collection}

We developed a semistructured interview guide using the theoretical domains framework (TDF), a broadly used integrative framework for identifying determinants of current or desired behaviors (Supplemental Appendix 1). ${ }^{19}$ Questions based on all but 1 TDF domain (behavioral regulation) were included at first. Because a primary aim was to learn about differences in PPI gastroprotection use across specialties, we included questions on current prescribing practices and perceived responsibility for PPI gastroprotection. We piloted the initial interview guide with 2 physicians. Because the guide did not change substantially after these pilot interviews, they were included in the final sample. Domains for which no relevant responses were obtained in early interviews (reinforcement, social influences, and intentions) were dropped after discussion at team meetings. All interviews were audio recorded and transcribed verbatim.

\section{Analysis}

An iterative, thematic analysis approach focusing on prescribing practices and barriers to the use of PPIs for gastroprotection, as well as similarities and differences across medical specialties, was used. Interview data were analyzed by carefully reading transcripts and applying codes and subcodes, organized around the TDF, to supporting quotes using NVivo software (QSR International). Additional codes were created for emergent themes. We performed analysis concurrently with ongoing interviews. Interviewers completed analytic memos $(n=15)$, along with preliminary interpretations, to summarize each interview. ${ }^{20}$ The team also kept procedural memos $(n=3)$ to document changes to the codebook, identify transcription errors, and track the number of times a topic was discussed by providers in each specialty. A detailed description of the multistep analytic and coding process, which included duplicate review of 8 transcripts, is available in Supplemental Appendix 2.

Team members then met to discuss which domains represented the most significant barriers to appropriate use of PPI gastroprotection according to the following 3 criteria: (1) each code's frequency, (2) evidence that respondents believed that constructs within each domain influenced the use of PPI gastroprotection, and (3) relevance to future intervention development based on behavior change theory. ${ }^{21}$ Two team members with formal academic training and experience in qualitative research (J.E.K., S.L.K.) directed interview guide and codebook development, data collection, and analysis. One of 3 study team members (J.E.K., D.H., S.L.K.) led all interviews, with up to 2 additional investigators present. This study was deemed exempt from review by the Institutional Review Board of the University of Michigan. Verbal consent was obtained from all interview participants, who were not compensated.

\section{RESULTS}

The participants included 5 PCPs, 4 cardiologists, 3 gastroenterologists, and 3 vascular surgeons. Demographic characteristics are shown in Table 1 . The majority of participants were male ( $n=10$ ), with 1 to 10 years of postfellowship medical practice experience (73.3\%).

Whereas respondents generally described limited use of PPI gastroprotection, there were notable differences in barriers within and across specialties. The most salient barriers to the use of PPI gastroprotection fell into the following 3 themes, which in some cases encompassed $>1$ TDF domain: 


\section{Table 1. Participant Characteristics}

\begin{tabular}{ll|ll}
\hline $\begin{array}{l}\text { Participants } \\
(\mathbf{n = 1 5 )}\end{array}$ & No. (\%) & $\begin{array}{l}\text { Participants } \\
(\mathbf{n = 1 5 )}\end{array}$ & No. (\%) \\
\hline Specialty & & Years of practice & \\
$\quad$ Primary care & $5(33.3)$ & $1-5$ & $5(33.3)$ \\
Cardiology & $4(26.7)$ & $6-10$ & $6(40.0)$ \\
Gastroenterology & $3(20)$ & $11-15$ & $1(6.7)$ \\
Vascular surgery & $3(20)$ & $16-20$ & $1(6.7)$ \\
Female & $5(33.3)$ & $21+$ & $2(13.3)$ \\
\hline
\end{tabular}

\section{Table 2. Supporting Quotes for PPI Prescribing Practices}

\section{Approach to Use of PPIs}

\section{Primary care}

[PCP 3] "I can't think of the last time I started [a PPI] for gastroprotection, but people tend to be on it already."

[PCP 2]

Interviewer: "So, how do you tend to use [PPIs] in your patients?"

Response: "GERD, GERD, and GERD. So, that's number 1. And number 2 is as an offset to nonsteroidal therapy."

[PCP 1] "I'm more likely thinking about can I take that PPI away than I am thinking about what can I start to prevent an ulcer?"

\section{Cardiology}

[Cardiologist 1] "For example, if I had a post- [ST-elevation myocardial infarction] patient that needed to be on aspirin and Plavix, and they had a big apical thrombus and they needed to be on Coumadin... and they are going to be on triple therapy for a month, then I would probably just put them on a PPI prophylactically, just because it's like definitely a higher bleeding risk to be on triple therapy..."

[Cardiologist 4]

Interviewer: "How often do you tend to prescribe it in patients who are asymptomatic just for the purposes of preventing bleeding?"

Response: "I can't think of times when I have done that."

[Cardiologist 3] "So, I put everybody I see and start on-even if they are on it, but I make sure everybody - if I have a patient who is on dual antiplatelet therapy, they go on a PPI. I pretty much - and I am super militant about this-and then, you know, I also don't like to continue [dual antiplatelet therapy] more than what I have to."

\section{Gastroenterology}

[Gastroenterologist 1] "I think, in general, PCPs or other physicians are pretty aware of the risk, and the patients, by the time they come to us, they are already on PPIs. So, I don't usually make-I don't usually start PPIs just for gastroprotection. I think it's more because I don't feel like I need to because they are already on it, typically."

[Gastroenterologist 2] "If somebody comes in to see me for a Gl problem, on my checklist is not to see if they are at risk for bleeding ulcer or complicated peptic ulcer disease. Now, if they bring to my attention any upper Gl symptoms, that will be a part of my evaluation, especially if it's peptic symptoms or, again, any reflux symptoms. But every patient that I see in clinic, I don't-you know, like in primary care they make sure somebody's mammogram is up to date. I don't look to see if a-I don't assess their risk for developing a bleeding ulcer and look to see if they are on appropriate gastroprotection."

\section{Vascular surgery}

[Vascular surgeon 3] "I generally do not prescribe them [PPIs] for my patients in an outpatient setting, so short of those people who get them in the perioperative setting, I am not initiating treatment for patients."

GERD = gastroesophageal reflux disease $; \mathrm{GI}=$ gastrointestinal; $\mathrm{PCP}=$ primary care physician; $\mathrm{PPI}=$ proton pump inhibitor; $\mathrm{ST}$ = section on an electrocardiogram between $\mathrm{S}$ wave and T wave.
(1) knowledge, including awareness of UGIB risk factors, nal rele, encompassing beliefs about how prescribing PPI gastrotection fit within perceived professional responsibilities. sccinctly encapsulate each specialty's prescribing pracThes, exemplar quotations are shown in Table 2. For each of plar quotations are embedded in the text below. Additional quotations are listed in Supplemental Tables 1-3.

\section{PPI Prescribing Practices}

Primary care physicians tended to use PPIs mostly for the treatment of symptoms (gastroesophageal reflux disease) rather than for gastroprotection. Even for patients using NSAIDs, which is a risk factor for both GI upset and UGIB, PPIs were more likely to be prescribed for the treatment of symptoms than for gastroprotection. Because of concerns regarding adverse effects, about which patients frequently inquired, PCPs focused more on deprescribing PPIs than on initiation for gastroprotection.

Cardiologists showed the greatest variation in use of PPI gastroprotection. Some prescribed PPIs for all patients on dual-antiplatelet therapy, some only for patients with certain risk factors (history of UGIB or use of an anticoagulant in addition to dual-antiplatelet therapy [triple therapy]), and some not at all.

Many gastroenterologists believed that a large proportion of their patients, regardless of UGIB risk, were already using PPIs before referral to them. They were unlikely to consider the need for gastroprotection unless it was related to the reason for consultation (eg, GI bleeding). One gastroenterologist was diligent about prescribing PPIs for patients on dual-antiplatelet therapy specifically, whereas the others tended to prescribe PPI gastroprotection haphazardly if they noticed multiple risk factors for UGIB.

Vascular surgeons rarely prescribed PPIs for clinic patients for gastroprotection or GI symptoms. They more often used PPIs for stress-ulcer prophylaxis in hospitalized patients. 


\section{Knowledge}

Knowledge of pharmacologic risk factors for UGIB varied by specialty. Cardiologists most readily identified the bleeding risks associated with antithrombotic drugs. Gastroenterologists and PCPs identified a broader range of risk factors, often mentioning NSAIDs but also Helicobacter pylori infection. Across specialties, there was broad awareness of prior UGIB as a risk factor for recurrence. However, few participants in any specialty mentioned increasing risk with age. Vascular surgeons' comments tended to consider risk factors associated with stress-ulcer bleeding among inpatients.

Most PCPs, cardiologists, and gastroenterologists believed that PPIs were at least somewhat effective for UGIB prevention. However, both across and within these specialties, clinicians varied in the perceived degree of effectiveness. Vascular surgeons were less knowledgeable about the effectiveness of PPI gastroprotection. A minority of physicians (1 gastroenterologist, 2 cardiologists) could identify specific guidelines on this topic. One of these cardiologists found the guideline lacking.

Cardiologist 2: They say really obvious stuff, like if you are adding an antiplatelet agent to an anticoagulant, the risk of bleeding is going to go up. And they talk about people that are at risk, you should consider it. And they don't define what "at risk" means. I mean, it's really a piece of junk. And it's very hard to read. It's very dense with sort of like summarizing studies and whatever but then, they don't give any conclusions.

Primary care physicians and gastroenterologists had the greatest awareness of possible adverse effects associated with PPIs (Table 3 ). Few nongastroenterologists recalled specific teaching about the use of PPIs for gastroprotection.

PCP 2: I cannot identify a specific time when I sat down, was taught or read about the ins and outs of PPIs. The learning that I have had in the last few years has mainly been about the long-term side effects... but the positive indication has been a much less prominent part of my learning about PPIs.

\section{Table 3. Participant Reference to Possible Adverse Effects Associated With PPI Use}

\begin{tabular}{lcccc}
\hline & $\begin{array}{c}\text { Primary } \\
\text { Care } \\
\text { Reference, No. (\%) }\end{array}$ & $\begin{array}{c}\text { Cardiology } \\
(\mathbf{n}=\mathbf{5})\end{array}$ & $\begin{array}{c}\text { Gastroenterology } \\
\mathbf{( n = 3 )}\end{array}$ & $\begin{array}{c}\text { Vascular } \\
\text { Surgery } \\
\mathbf{( n = 3 )}\end{array}$ \\
\hline $\begin{array}{l}\text { Any mention of possible } \\
\text { adverse effects }\end{array}$ & $5(100)$ & $2(50)$ & $3(100)$ & $1(33.3)$ \\
$\begin{array}{l}\text { Clostridioides difficile or } \\
\text { other enteric infection }\end{array}$ & $4(80)$ & $1(25)$ & $1(33.3)$ & $1(33.3)$ \\
$\begin{array}{l}\text { Dementia } \\
\text { Pneumonia }\end{array}$ & $4(80)$ & $0(0)$ & $3(100)$ & $0(0)$ \\
$\begin{array}{l}\text { Chronic kidney disease } \\
\text { Bone disease }\end{array}$ & $3(60)$ & $2(50)$ & $1(33.3)$ & $1(33.3)$ \\
\hline PPI = proton pump inhibitor. & $3(60)$ & $1(25)$ & $2(66.7)$ & $0(0)$ \\
\hline
\end{tabular}

\section{Decision Processes}

Primary care physicians generally relied on a gestalt rather than a systematic approach to identify high-risk patients. Concerns regarding adverse effects factored prominently in their decisions on PPI gastroprotection. Primary care physicians sometimes felt conflicted about using NSAIDs at all because of reluctance to prescribe gastroprotection, leaving fewer options for treating pain.

PCP 2: The thing that worries me in my head is the patients who chronically or intermittently use NSAIDs. How big is their risk of upper GI bleeding, especially as they age? Where do I draw the balance between giving them NSAIDs for their many, many musculoskeletal complaints for which NSAIDs provide significant relief and warn them not to do this, you know, not to take NSAIDs and suffer a little bit more because I worry about them, versus PPI? There's a whole other set of concerns around chronic use of PPIs.

Cardiologists often thought about bleeding risk (from any anatomic site) when considering the use of antithrombotic drugs. Perceived bleeding risk sometimes affected the choice of coronary stents, antithrombotic medications, and duration of treatment. Gastrointestinal bleeding was perceived to be relatively uncommon and less severe than intracranial bleeding.

Cardiologist 4: I want to get to the point about, okay, these [antithrombotic drugs] are not without risk. Probably, I will often mention that you can bleed in the GI tract, but the thing that I feel that person really, really, really needs to know is they might also bleed in their brain and that could alter their life.

Cardiologists perceived the risk of PPI adverse effects to be minor, factoring minimally into decision making. They perceived the greater downside of PPI gastroprotection to be increased pill burden and cost.

Cardiologist 1: It's like an out-of-pocket expense and, like, the more medications you prescribe to a patient, like, they have a perception that means they are sicker. And then cardiology, we use cocktails of medications, so I guess what I am saying is, you need to have more reason to prescribe it... or the patient who doesn't have history of GI bleeding, like, what is the data to support prescribing it in addition to what they are already taking?

One cardiologist believed that guidelines, especially if based on expert opinion, had little effect on clinical decisions, but that clinical trial data or the opinion of a trusted colleague could be persuasive. When gastroenterologists identified patients they thought were at high risk for bleeding, they tended to believe that the benefits of PPIs outweighed the risks; the decision-making process involved less ambiguity. Vascular 
surgeons perceived GI bleeding to be rare and attempted to minimize this risk by avoiding particularly high-risk drug combinations, especially triple therapy. Because they seldom prescribed gastroprotection, little information was elicited on decision making.

\section{Professional Role}

Primary care physicians felt that gastroprotection was often a low priority during the limited time of a clinic visit, which could include symptomatic complaints and preventive care.

PCP 3: ... a patient walks in for a sore throat, and...the BPA [Best Practice Advisory] is going to come up that they are due for their mammogram, their foot exam, their eye exam, their colonoscopy and, oh, by the way, their pneumonia vaccine, so it's highly unlikely by the time I get through talking about their sore throat and try to manage some of that that I have even any brain power left to be thinking about some of these things.

Gastroenterologists and vascular surgeons also felt that gastroprotection was overlooked among competing issues and was not routinely considered. However, cardiologists felt that bleeding risk reduction was part of the whole package of clinical care that they should provide when treating patients with antithrombotic drugs, even if they were not accustomed to prescribing gastroprotection.

Cardiologist 3: If I am putting a patient on a medication that is potentially increasing their risk of bleeding, then it's my responsibility to do whatever I can to fix it. Now, I think it is completely reasonable for me to say I'm not going to put you on a PPI because I don't feel comfortable putting you on a PPI. I would rather consult my colleague in GI. But I don't want to delegate the responsibility to a primary care provider, who then has to go to a third specialist.

Most PCPs felt that ideally, the clinician prescribing a high-risk drug, frequently a specialist, should be responsible for considering gastroprotection because it could otherwise fall through the cracks. Nonetheless, they still felt ultimate responsibility for ensuring that their patients received appropriate care. Whereas gastroenterologists believed that gastroprotection should be a priority, they perceived that a large proportion of their clinic patients already used PPIs for symptomatic GI conditions, and they rarely received referrals for consideration of gastroprotection. Some gastroenterologists felt that PCPs were already attending to it.

Interviewer: Do you feel like this is something that falls under the province of gastroenterology to, like, think about the use of PPIs for preventive purposes...or not?

Gastroenterologist 1: I don't know that it needs to be done by a gastroenterologist. I feel like...PCPs and any internist, you know, should have probably a bead on that.

One vascular surgeon expressed the opinion that it was "dealer's choice" which clinician involved in a patient's care prescribed gastroprotection, another felt that PCPs should be primarily responsible, and a third felt that perhaps the vascular surgeon, if prescribing a high-risk drug, should be responsible.

\section{DISCUSSION}

This qualitative study explored prescribing practices and barriers to the use of PPI gastroprotection among PCPs, cardiologists, gastroenterologists, and vascular surgeons, all of whom see patients appropriate for this guideline-based practice. The most salient barriers fell into the following 3 themes, which can operate differentially across specialties: (1) knowledge, (2) decision processes, and (3) professional role. Across specialties, few clinicians could specify patients for whom PPI gastroprotection would be appropriate. Primary care physicians in particular expressed reservations about using PPIs because of concerns regarding adverse effects. Primary care physicians, gastroenterologists, and vascular surgeons all felt that consideration of gastroprotection took a low priority. In contrast, cardiologists felt that it was important to address the risk of hemorrhage, including from the GI tract, when prescribing antithrombotic drugs. Finally, no single specialty owned the issue of gastroprotection. Some gastroenterologists, vascular surgeons, and cardiologists felt that gastroprotection was something that PCPs did or ought to manage. However, many PCPs felt that if a specialist prescribed a high-risk drug, the same clinician should prescribe gastroprotection. These barriers might in large part explain why PPI gastroprotection is underused.

Few studies have investigated barriers to or the role of medical specialty in prescribing PPI gastroprotection. Consistent with our present findings, one study that used chart review found that gastroprotection was poorly recognized as an indication for PPI prescribing, except among rheumatologists. ${ }^{22}$ In a separate survey study, PCPs had high levels of awareness of possible PPI adverse effects, which was an impetus to discontinue PPIs even in the case of a patient at high risk for upper GI bleeding. ${ }^{17}$ The present study adds to the existing literature by providing a holistic examination of prescribing practices for gastroprotection. Importantly, we found barriers affecting providers' capability, opportunity, and motivation to prescribe gastroprotection (namely insufficient knowledge, time, and prioritization), which have been identified as the 3 preconditions needed to perform a behavior. ${ }^{21}$ Most often, providers faced $>1$ of these barriers.

Our finding of unclear delegation of responsibility between PCPs and specialists highlights the common challenge of coordination of care. In a 2019 survey, a substantial proportion of PCPs in the United States indicated that they failed to receive information that they needed for ongoing patient care, or timely communication from specialists, ${ }^{23} \mathrm{a}$ sentiment echoed in focus groups with PCPs and specialists about coordination of care in the Veterans Affairs system. ${ }^{24}$ Ultimately, coordinated care requires shared understanding and knowledge between clinicians. ${ }^{25}$ Thus, the disparate 
levels of knowledge that we found regarding gastroprotection are a clear impediment.

The present study has several limitations. First, the sample included 15 purposively sampled clinicians from 4 medical specialties within a single health system. It was not our intent to generalize to all clinicians in each specialty or to other health systems. Rather, the sampling strategy was intended to illustrate the heterogeneity of perspectives on gastroprotection, with a focus on the role of medical specialty. Second, we interviewed a relatively small number of gastroenterologists and vascular surgeons. Whereas it is possible that additional interviews in these specialties would have identified further variation in practice, we believe the major themes were identified, given our finding of early data saturation. Third,

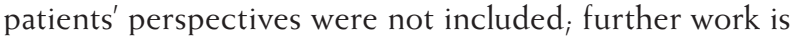
planned on this factor.

The above limitations notwithstanding, our present findings can inform future efforts to improve prescribing of PPI gastroprotection. Complex interventions that address $>1$ barrier will likely be necessary. Indeed, several studies that have tested multicomponent interventions involving professional education, incentive payments, provider feedback, and pharmacist support have effectively decreased the proportion of high-risk patients without gastroprotection (odds ratios 0.55-0.72). ${ }^{26-28}$ The ability to implement and sustain such intense interventions in a fragmented US health care system is unclear.

Several other strategies, alone or in combination, might be worth investigating. To improve coordination of care, better guidelines are needed. In the past, guidance statements have been published mostly in cardiology and gastroenterology journals and have focused on discrete high-risk groups such as patients using NSAIDs ${ }_{1}{ }^{12}$ aspirin, ${ }^{10}$ or dual-antiplatelet therapy. ${ }^{7,8}$ Guidelines should be comprehensive and target PCPs and specialists at once, similar to US Preventive Services Task Force guidelines. They should also specify who should prescribe gastroprotection and when (eg, cardiologists after placing a coronary stent), which is often overlooked in guidelines. ${ }^{29}$ Institutional service agreements could serve a similar function. Coordination of care could also benefit from a population health management approach, characterized by systems to identify high-risk patients, share information among providers, and intervene when necessary, typically using a care coordinator. Clinical pharmacist practitioners, who work alongside physicians and who can in some circumstances independently prescribe medications, could be incorporated in this approach, minimizing the need for provider involvement. ${ }^{30}$ To increase prioritization, patients could be activated to bring up gastroprotection with their providers. ${ }^{31}$ Pragmatically, publicly reported quality measures related to PPI gastroprotection would likely create greater motivation. Electronic health record alerts have proven minimally effective for gastroprotection. ${ }^{32}$

Our finding that PCPs are disproportionately focused on deprescribing PPIs underscores the fact that a reappraisal of PPIs is overdue. Contrary to what many clinicians believe, a causal role for PPIs has not been established for most of the conditions with which they have been associated. ${ }^{16} \mathrm{~A}$ randomized 3-year trial of pantoprazole vs placebo for gastroprotection evaluated numerous possible adverse effects over 53,000 person-years of follow-up and provides the best available data on this topic. ${ }^{33,34}$ Proton pump inhibitors were associated with an increased risk only of enteric infections, with a number needed to harm of $>300$. It should be emphasized that for patients at high risk of UGIB, the benefits of PPI gastroprotection outweigh the small risk of adverse effects. ${ }^{35}$ Furthermore, stopping a PPI in the wrong patient can cause serious injury. ${ }^{36}$

In conclusion, clinicians in 4 medical and surgical specialties each faced distinct sets of barriers to the use of PPI gastroprotection, related to knowledge, decision processes, and professional role. Multicomponent interventions will likely be required to improve appropriate use, and strategies that do not depend entirely on clinicians, including pharmacist facilitation and patient activation, are worth investigating.

\section{Read or post commentaries in response to this article.}

Key words: proton pump inhibitors; gastroprotection; gastrointestinal bleeding; prevention; barriers

Submitted September 15, 2020; submitted, revised, February 18, 2021; accepted March 30, 2021.

Author contributions: J.E.K.: study concept and design, conduct interviews, analysis and interpretation of data, drafting of the manuscript, and critical revision of the manuscript for important intellectual content. D.H.: study design, conduct interviews, analysis and interpretation of data, drafting of the manuscript, and critical revision of the manuscript for important intellectual content. A.N.K.: conduct interviews, analysis and interpretation of data, and critical revision of the manuscript for important intellectual content. C.R.R., R.D.V., S.D.S.: study concept and design and critical revision of the manuscript for important intellectual content. S.L.K.: study concept and design, conduct interviews, analysis and interpretation of data, and critical revision of the manuscript for important intellectual content.

Funding support: This study was funded by the National Institute of Diabetes and Digestive and Kidney Diseases with a K23 award (K23DK118179) (J.E.K.), US Department of Veterans Affairs (VA) Health Services Research and Development Service (HSRD) Research Career Scientist award (HSRD RCS 11-222) (S.L.K.), and VA HSRD Service Directed Research award (SDR 101 HX002693-01) (J.E.K., S.D.S.). The funders had no role in study design, data collection and analysis, decision to publish, or preparation of the manuscript. The views expressed in this manuscript do not represent the views of the VA administration or $\mathrm{NIH}$.

\section{Supplemental materials}

\section{References}

1. Peery AF, Crockett SD, Murphy CC, et al. Burden and cost of gastrointestinal, liver, and pancreatic diseases in the United States: update 2018. Gastroenterology. 2019;156(1):254-272.e11. 10.1053/j.gastro.2018.08.063

2. Abougergi MS, Travis AC, Saltzman JR. The in-hospital mortality rate for upper GI hemorrhage has decreased over 2 decades in the United States: a nationwide analysis. Gastrointest Endosc. 2015;81(4):882-888.e1. 10.1016/j. gie.2014.09.027

3. Wuerth BA, Rockey DC. Changing epidemiology of upper gastrointestinal hemorrhage in the last decade: a nationwide analysis. Dig Dis Sci. 2018; 63(5):1286-1293. 10.1007/s10620-017-4882-6

4. García Rodríguez LA, Lin KJ, Hernández-Díaz S, Johansson S. Risk of upper gastrointestinal bleeding with low-dose acetylsalicylic acid alone and in combination with clopidogrel and other medications. Circulation. 2011; 123(10):1108-1115. 10.1161/CIRCULATIONAHA.110.973008 
5. Budnitz DS, Lovegrove MC, Shehab N, Richards CL. Emergency hospitalizations for adverse drug events in older Americans. N Engl J Med. 2011; 365(21):2002-2012. 10.1056/NEJMsa1103053

6. Scally B, Emberson JR, Spata E, et al. Effects of gastroprotectant drugs for the prevention and treatment of peptic ulcer disease and its complications: a meta-analysis of randomised trials. Lancet Gastroenterol Hepatol. 2018;3(4): 231-241. 10.1016/S2468-1253(18)30037-2

7. Valgimigli M, Bueno H, Byrne RA, et al; ESC Scientific Document Group: ESC Committee for Practice Guidelines (CPG); ESC National Cardiac Societies. 2017 ESC focused update on dual antiplatelet therapy in coronary artery disease developed in collaboration with EACTS: The Task Force for dual antiplatelet therapy in coronary artery disease of the European Society of Cardiology (ESC) and of the European Association for Cardio-Thoracic Surgery (EACTS). Eur Heart J. 2018;39(3):213-260. 10.1093/eurheartj/ehx419

8. Levine GN, Bates ER, Blankenship JC, et al. 2011 ACCF/AHA/SCAI Guideline for Percutaneous Coronary Intervention: a report of the American College of Cardiology Foundation/American Heart Association Task Force on Practice Guidelines and the Society for Cardiovascular Angiography and Interventions. Circulation. 2011;124(23):e574-e651. 10.1161/CIR.0b013e31823ba622

9. Barkun AN, Almadi M, Kuipers EJ, et al. Management of nonvariceal upper gastrointestinal bleeding: guideline recommendations from the International Consensus Group. Ann Intern Med. 2019;171(11):805-822. 10.7326/M19-1795

10. Bhatt DL, Scheiman J, Abraham NS, et al; American College of Cardiology Foundation Task Force on Clinical Expert Consensus Documents. ACCF/ACG/ AHA 2008 expert consensus document on reducing the gastrointestinal risks of antiplatelet therapy and NSAID use: a report of the American College of Cardiology Foundation Task Force on Clinical Expert Consensus Documents. Circulation. 2008;118(18):1894-1909. 10.1161/CIRCULATIONAHA.108.191087

11. Abraham NS, Hlatky MA, Antman EM, et al; ACCF/ACG/AHA. ACCF/ACG/AHA 2010 expert consensus document on the concomitant use of proton pump inhibitors and thienopyridines: a focused update of the ACCF/ACG/AHA 2008 expert consensus document on reducing the gastrointestinal risks of antiplatelet therapy and NSAID use. Am J Gastroenterol. 2010;105(12):2533-2549. 10.1038/ajg.2010.445

12. Lanza FL, Chan FKL, Quigley EMM; Practice Parameters Committee of the American College of Gastroenterology. Guidelines for prevention of NSAID-related ulcer complications. Am J Gastroenterol. 2009;104(3):728-738. 10.1038/ajg.2009.115

13. Kumbhani DJ, Cannon CP, Beavers CJ, et al. ACC expert consensus decision pathway for anticoagulant and antiplatelet therapy in patients with atrial fibrillation or venous thromboembolism undergoing percutaneous coronary intervention or with atherosclerotic cardiovascular disease. J Am Coll Cardiol. 2021;77(5):629-658. 10.1016/j.jacc.2020.09.011

14. Kanno T, Moayyedi P. Who needs gastroprotection in 2020? Curr Treat Options Gastroenterol. 2020;18(4):1-17. 10.1007/s11938-020-00316-9

15. Medlock S, Eslami S, Askari M, et al. Co-prescription of gastroprotective agents and their efficacy in elderly patients taking nonsteroidal anti-inflammatory drugs: a systematic review of observational studies. Clin Gastroenterol Hepatol. 2013;11(10):1259-1269.e10. 10.1016/j.cgh.2013.05.034

16. Vaezi MF, Yang YX, Howden CW. Complications of proton pump inhibitor therapy. Gastroenterology. 2017;153(1):35-48. 10.1053/j.gastro.2017.04.047

17. Kurlander JE, Rubenstein JH, Richardson CR, et al. Physicians' perceptions of proton pump inhibitor risks and recommendations to discontinue: a national survey. Am J Gastroenterol. 2020;115(5):689-696. 10.14309/ajg. 0000000000000558

18. Creswell JW. A Concise Introduction to Mixed Methods Research. $1^{\text {st }}$ edition. SAGE Publications, Inc; 2014.

19. Cane J, O'Connor D, Michie S. Validation of the theoretical domains framework for use in behaviour change and implementation research. Implement Sci. 2012;7:37. 10.1186/1748-5908-7-37
20. Birks M, Chapman Y, Francis K. Memoing in qualitative research: probing data and processes. J Res Nurs. 2008;13(1):68-75. 10.1177/1744987107081254

21. Michie S, van Stralen MM, West R. The behaviour change wheel: a new method for characterising and designing behaviour change interventions. Implement Sci. 2011;6:42. 10.1186/1748-5908-6-42

22. Dries AM, Richardson P, Cavazos J, Abraham NS. Therapeutic intent of proton pump inhibitor prescription among elderly nonsteroidal anti-inflammatory drug users. Aliment Pharmacol Ther. 2009;30(6):652-661. 10.1111/ j.1365-2036.2009.04085.x

23. Doty MM, Tikkanen R, Shah A, Schneider EC. Primary care physicians' role in coordinating medical and health-related social needs in eleven countries. Health Aff (Millwood). 2020;39(1):115-123. 10.1377/hlthaff.2019.01088

24. Kim B, Lucatorto MA, Hawthorne K, et al. Care coordination between specialty care and primary care: a focus group study of provider perspectives on strong practices and improvement opportunities. J Multidiscip Healthc. 2015;8:47-58. 10.2147/JMDH.S73469

25. Gittell JH, Godfrey M, Thistlethwaite J. Interprofessional collaborative practice and relational coordination: improving healthcare through relationships. J Interprof Care. 2013;27(3):210-213. 10.3109/13561820.2012.730564

26. Avery AJ, Rodgers S, Cantrill JA, et al. A pharmacist-led information technology intervention for medication errors (PINCER): a multicentre, cluster randomised, controlled trial and cost-effectiveness analysis. Lancet. 2012; 379(9823):1310-1319. 10.1016/S0140-6736(11)61817-5

27. Dreischulte T, Donnan P, Grant A, Hapca A, McCowan C, Guthrie B. Safer prescribing--a trial of education, informatics, and financial incentives. $N$ Engl J Med. 2016;374(11):1053-1064. 10.1056/NEJMsa1508955

28. Guthrie B, Kavanagh K, Robertson C, et al. Data feedback and behavioural change intervention to improve primary care prescribing safety (EFIPPS): multicentre, three arm, cluster randomised controlled trial. BMJ. 2016;354: i4079. 10.1136/bmj.i4079

29. Michie $S$, Johnston $M$. Changing clinical behaviour by making guidelines specific. BMJ. 2004;328(7435):343-345. 10.1136/bmj.328.7435.343

30. Scott MA, Heck JE, Wilson CG. The integral role of the clinical pharmacist practitioner in primary care. N C Med J. 2017;78(3):181-185. 10.18043/ncm. 78.3.181

31. Wallis KA, Elley CR, Lee A, Moyes S, Kerse N. Safer Prescribing and Care for the Elderly (SPACE): protocol of a cluster randomized controlled trial in primary care. JMIR Res Protoc. 2018;7(4):e109. 10.2196/resprot.9839

32. Gill JM, Mainous AG III, Koopman RJ, et al. Impact of EHR-based clinical decision support on adherence to guidelines for patients on NSAIDs: a randomized controlled trial. Ann Fam Med. 2011;9(1):22-30. 10.1370/afm.1172

33. Moayyedi P, Eikelboom JW, Bosch J, et al; COMPASS Investigators. Safety of proton pump inhibitors based on a large, multi-year, randomized trial of patients receiving rivaroxaban or aspirin. Gastroenterology. 2019;157(3):682691.e2. 10.1053/j.gastro.2019.05.056

34. Corley DA. Safety and complications of long-term proton pump inhibitor therapy: getting closer to the truth. Gastroenterology. 2019;157(3):604-607. 10.1053/j.gastro.2019.07.039

35. Freedberg DE, Kim LS, Yang YX. The risks and benefits of long-term use of proton pump inhibitors: expert review and best practice advice from the American Gastroenterological Association. Gastroenterology. 2017;152(4):706715. 10.1053/j.gastro.2017.01.031

36. Platt KD, Saini SD, Kurlander JE. Selecting the appropriate patients for proton pump inhibitor discontinuation: a teachable moment. JAMA Intern Med. 2019;179(9):1276-1277. 10.1001/jamainternmed.2019.2382 